\title{
Caspase-3 inhibitor prevents the apoptosis of brain tissue in rats with acute cerebral infarction
}

\author{
YUHUA SUN ${ }^{1,2}$, YUMING XU ${ }^{2}$ and LIJIAO GENG ${ }^{1}$ \\ ${ }^{1}$ Department of Neurology, Henan University Huaihe Hospital, Kaifeng, Henan 475001; \\ ${ }^{2}$ Department of Neurology, The First Affiliated Hospital of Zhengzhou University, Zhengzhou, Henan 450052, P.R. China
}

Received July 27, 2014; Accepted March 27, 2015

DOI: $10.3892 /$ etm.2015.2462

\begin{abstract}
The aim of the present study was to investigate the effect of the caspase-3 inhibitor z-DEVD-fmk on the apoptosis of the brain tissues of rats with acute cerebral infarction. Middle cerebral artery occlusion was used to establish a rat model of infarction, and the rats were randomly divided into a sham group $(n=15)$, model group $(n=15)$ and treatment group $(\mathrm{n}=15)$. $\mathrm{z}$-DEVD-fmk $(2.5 \mu \mathrm{g} / \mathrm{kg})$ was injected into the intracranial artery of rats in the treatment group, while the same volume of phosphate-buffered saline solution was administered to the rats of the sham and model groups. After $48 \mathrm{~h}$, all rats were sacrificed and their brain tissues were removed. The caspase- 3 mRNA level, protein level and activity, brain cell apoptosis index and infarction scope of the three groups were analyzed. Neurological impairment was also assessed. At $48 \mathrm{~h}$ after model establishment, the caspase-3 mRNA and protein levels in the brain tissues of the model group were significantly higher than those of the sham group, and those in the treatment group were significantly lower than those in the model group $(\mathrm{P}<0.05)$; however, they remained significantly higher than those in the sham group. Caspase-3 activity in the model group was significantly higher than that in the sham group, and treatment with the caspase- 3 inhibitor significantly reduced caspase- 3 activity compared with that in the model group $(\mathrm{P}<0.05)$. The apoptosis index and infarction scope in the model and treatment groups were significantly increased compared with those in the sham group, and were significantly lower in the treatment group than in the model group $(\mathrm{P}<0.05)$. The neurological impairment of rats in the model and treatment groups was increased significantly compared with that in the sham group, and the treatment group exhibited a significantly lower level of neurological impairment than the model group $(\mathrm{P}<0.05)$. In conclusion, the caspase-3 inhibitor z-DEVD-fmk effectively inhibited apoptosis and delayed the necrosis of
\end{abstract}

Correspondence to: Professor Yuming $\mathrm{Xu}$, Department of Neurology, The First Affiliated Hospital of Zhengzhou University, 1 Jianshe Road, Zhengzhou, Henan 450052, P.R. China

E-mail: yumingxu@yeah.net

Key words: acute cerebral infarction, caspase-3 inhibitor, apoptosis brain tissue cells in rats with acute cerebral infarction, and had certain protective effects on brain tissue.

\section{Introduction}

Acute cerebral infarction is one of the most common clinical cardiovascular and cerebrovascular diseases. Due to its high incidence and fatality rate, it has become one of the most serious diseases affecting human health $(1,2)$. The pathological basis of acute cerebral infarction is the disruption of blood supply in the brain, with ischemia and hypoxia causing ischemic necrosis and cerebral malacia in focal brain tissue (3). In the acute stage of cerebral infarction, vascular occlusion can lead to a reduction in blood flow in the affected region and create a hypoxic state; cerebral hypoxia and recanalization cause a stress response, leading to the release of a large number of free radicals and the stimulation of brain cells, causing neuronal cell apoptosis that ultimately results in a loss of biological function $(4,5)$. Therefore, apoptosis plays an important role in the development process of acute cerebral infarction.

A large number of studies have indicated that the apoptosis of brain cells and neurons in rats and other animal models with acute cerebral infarction is significantly increased (6). Apoptosis is a cascade reaction mediated by cysteine proteinases (caspases). These include caspase-3, a protein that is affected by the cell surface death receptor-mediated and mitochondrial apoptosis pathways and thus plays an important role in the mediation of cell apoptosis (7-9). Studies have shown that caspase-3 expression levels and protein activity are significantly increased in the brain cells of patients with acute cerebral infarction, and there is a certain correlation with the prognosis of the patient (10). Therefore, the effective delay and inhibition of brain cell or neuronal apoptosis in patients with acute cerebral infarction reduces brain tissue damage and necrosis, decreases the fatality and disability rates, and has a protective effect on the brain. At present, acute cerebral infarction is usually treated by anti-free radical, anti-oxidation and thrombolytic therapies, among others, and there are very few reports concerning the direct use of anti-apoptotic drugs for the treatment of acute cerebral infarction (11). Therefore, the present study explored the effect of the caspase-3 inhibitor z-DEVD-fmk on the apoptosis of brain tissue in rats with acute cerebral infarction, with the aim of identifying a new treatment option for the clinical treatment of acute cerebral infarction. 


\section{Materials and methods}

Establishment of the acute cerebral infarction model. Sprague-Dawley rats (180-200 g) were purchased from the Shanghai Laboratory Animal Center (SLAC, Shanghai, China). The method of Longa et al was adopted to establish a rat model of middle cerebral artery infarction (12). At $2 \mathrm{~h}$ after the surgery, rats showed listlessness, homolateral Horner's syndrome, contralateral forelimb prolapse, internal adduction and rotation, and spontaneously homolateral circling, indicating the successful establishment of model. If there were no such symptoms, then the establishment of model was considered to have failed, and the rat was removed from the study.

The rats were divided into three groups, specifically the sham group $(\mathrm{n}=15)$, model group $(\mathrm{n}=15)$ and treatment group $(n=15)$. Models were established according to the literature method (12) for the model and treatment groups. In the sham group, the vasculature of the rats was separated, but occlusion of the artery by suture was not conducted. At $3 \mathrm{~h}$ after the successful establishment of the model in the treatment group, an intravenous injection of the caspase-3 inhibitor z-DEVD-fmk (2.5 $\mu \mathrm{g} / \mathrm{kg}$; BioVision, Milpitas, CA, USA) was injected immediately via the tail vein. The same volume of phosphate-buffered saline (PBS) solution was injected into the tail veins of rats in the model and sham groups. After $48 \mathrm{~h}$, rats were sacrificed and analyses were conducted. This study was carried out in strict accordance with the recommendations in the Guide for the Care and Use of Laboratory Animals of the National Institutes of Health (Eighth Edition, 2012; Bethesda, MD, USA). The animal use protocol has been reviewed and approved by the Institutional Animal Care and Use Committee (IACUC) of the First Affiliated Hospital of Zhengzhou University (Zhengzhou, China).

Analysis of neurological function. The Bederson scale was adopted to analyze the neurological impairment of rats in the sham, model and treatment groups at 12,24 and $48 \mathrm{~h}$, respectively, after surgery (13). The scores were as follows: 0 , no symptoms of neurological deficit; 1 , unable to fully extend the right front paw; 2 , circling to the left; 3 , toppling over to one side; 4 , loss of spontaneous walking and loss of consciousness.

Reverse transcription-quantitative polymerase chain reaction $(R T-q P C R)$. Total RNA was extracted from brain tissues according to instructions of the RNA extraction kit (Invitrogen Life Technologies, Carlsbad, CA, USA). Following the extraction and purification of the RNA, a spectrophotometer was used to measure the concentration of RNA. The RNA was transcribed into cDNA with the use of reverse transcription reagent (Takara Biotechnology Co., Ltd., Dalian, China). PCR primers for rat caspase-3 were designed, with sequences as follows: forward, 5'-GGTATTGAGACAGACAGTGG-3' and reverse, 5'-CATGGGATCTGTTTCTTTGC-3'. Then, the qPCR reaction mixture was prepared as follows: $10 \mu 12 \mathrm{X}$ SYBR Green Universal qPCR Master Mix (Roche, Basel, Switzerland), upstream/downstream primers (10 $\mu \mathrm{mol} / \mathrm{l} ; 1 \mu \mathrm{l}$ of each) and $1 \mu \mathrm{l} \mathrm{cDNA}$, with double-distilled water to a final volume of $20 \mu \mathrm{l}$. The volume prepared was adjusted according to the required number of test samples, and $20 \mu 1$ was the volume used in each hole of the PCR plate. The reaction mixture was centrifuged for $10 \mathrm{~min}$ at $2,000 \mathrm{x} \mathrm{g}$. PCR was carried out according to the following reaction conditions: Pre-denaturation at $95^{\circ} \mathrm{C}$ for $30 \mathrm{sec}$; denaturation at $95^{\circ} \mathrm{C}$ for $3 \mathrm{sec}$; and annealing extension at $60^{\circ} \mathrm{C}$ for $30 \mathrm{sec}$; with the generation of dissociation curves. Finally, the data were directly provided by the real-time fluorescent quantitative PCR instrument (Bio-Rad Laboratories, Inc., Hercules, CA, USA).

Western blot assay. In this assay, $1 \mathrm{~g}$ rat brain tissue was placed in a mortar filled with liquid nitrogen, and was ground to a powder. To the powdered tissue was added $300 \mu 1$ cell lysis buffer (Wuhan Boster Biological Technology, Ltd., Wuhan, China) and $3 \mu \mathrm{l}$ protease inhibitor, and the mortar was placed on ice for $30 \mathrm{~min}$. The mixture was then centrifuged at $15,000 \mathrm{xg}$ for $15 \mathrm{~min}$. The supernatant was taken in order to measure the protein concentration. A $4 \mathrm{X}$ sample buffer solution was added to the sample, which was then boiled for $30 \mathrm{~min}$ followed by centrifugation. SDS-PAGE was conducted to transfer the protein to polyvinylidene difluoride membranes. Following blocking with 5\% skimmed milk, the membrane was incubated with primary polyclonal goat anti-caspase-3 antibody (1:100; sc-189; Santa Cruz Biotechnology, Inc., Santa Cruz, CA, USA) overnight at $37^{\circ} \mathrm{C}$. The membrane was then washed with PBS with Tween 20 (PBST) three times, and then incubated with horseradish peroxide-coupled sheep anti-mouse secondary antibody (Beijing Kangwei Technology Group Co., Ltd., Beijing, China) at room temperature for $1 \mathrm{~h}$. After washing with PBST three times, chemiluminescent reagent (GS009; Beyotime Institute of Biotechnology, Shanghai, China) was added to the blots and images were captured. $\beta$-actin was also measured as an internal control, and gray-scale computing software (Tocan, Inc., Shanghai, China) was used to analyze the protein band intensities and calculate the relative expression of the target protein.

Caspase-3 activity assay. This was carried in accordance with the instructions provided with the caspase- 3 protein activity detection reagent (Beyotime Institute of Biotechnology, Beijing, China).

Terminal deoxynucleotidyl transferase-mediated deoxyuridine triphosphate nick end labeling (TUNEL) staining. Antigen retrieval was conducted according to the equipment manufacturer's instructions (Roche) and Following dewaxing, $20-\mu \mathrm{m}$ rat brain sections from each group were washed with PBST three times. The TUNEL staining procedure was executed in strict accordance with the instructions provided with the TUNEL kit (Roche). The specimens were observed at a magnification of $x 400$, and 10 visual fields of the infarct zone and peripheral area were randomly selected. In each field, the proportion of apoptotic cells among all cells was calculated as the apoptotic index of the brain cells.

2,3,5-Triphenyltetrazolium chloride (TTC) analysis. Following sacrifice, brain tissues were extracted from the mice, the olfactory bulb, cerebellum and brain stem were removed, and the 

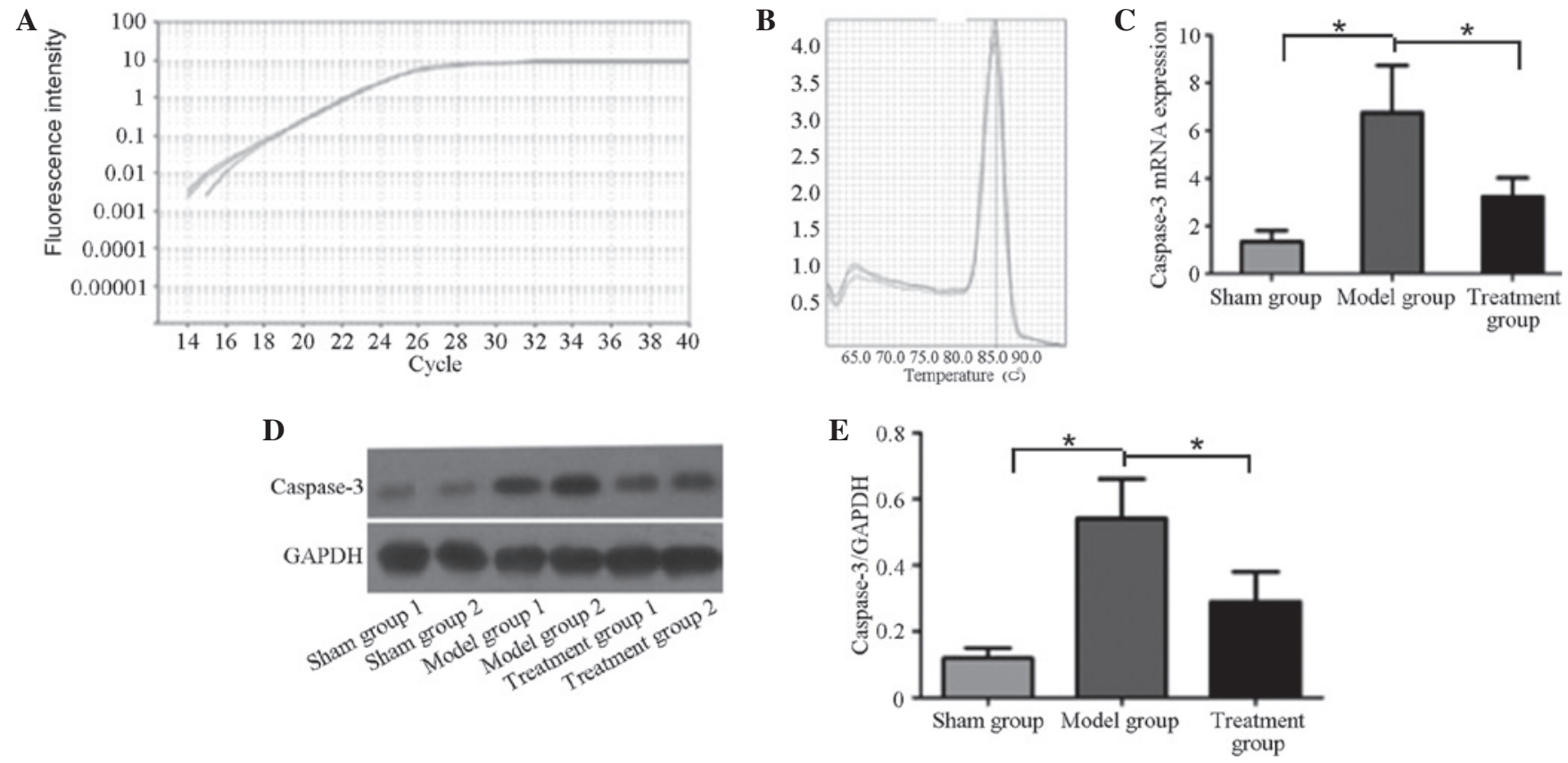

Figure 1. Comparison of caspase-3 mRNA and protein expression in the three groups. (A) Amplification curve and (B) melting curve obtained during caspase-3 amplification by qPCR. (C) Caspase-3 mRNA levels in the sham, model and treatment groups. (D) Western blotting and (E) quantitative analysis of caspase-3 protein levels in the sham, model and treatment groups. qPCR, quantitative polymerase chain reaction.

A

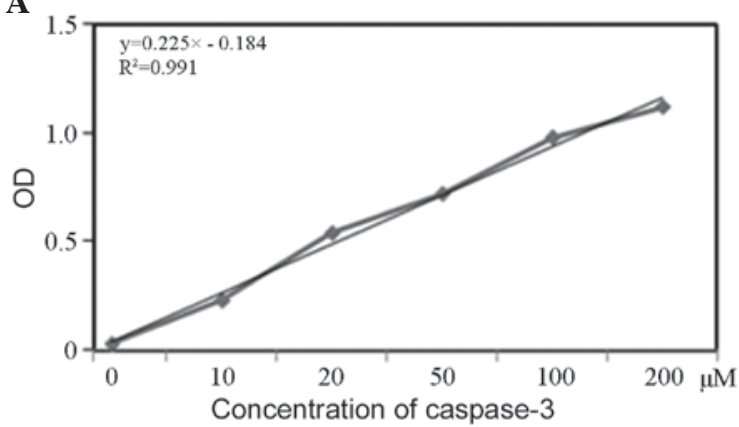

B

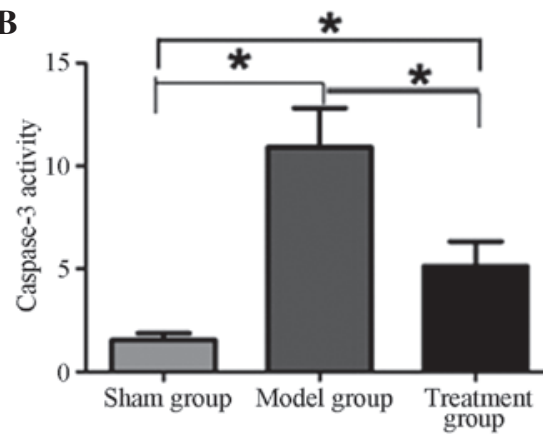

Figure 2. Comparison of caspase-3 activity in the three groups. (A) Standard curve of caspase-3 enzyme activity; (B) Caspase-3 activity in sham, model and treatment groups. ${ }^{*} \mathrm{P}<0.05$.

remaining tissue was cut into coronal slices. The slices were put into $2 \%$ TTC phosphate buffer solution (Sigma-Aldrich, St. Louis, MO, USA) at $37^{\circ} \mathrm{C}$ for staining for $20 \mathrm{~min}$. Then, paraformaldehyde was used to fix the tissue slices. The normal tissue was stained red and the infarcted tissue was white. The infarcted tissues were separated from the normal tissues under a microscope, and an analytical balance was used to weigh the wet normal tissues and infarcted tissues. The infarction scope was calculated as the weight of the infarcted tissues as a percentage of the total weight of infarcted tissues plus normal brain tissues.

Statistical analysis. All data were analyzed using SPSS software, version 17.0 (SPSS, Inc., Chicago, IL, USA). Measurement data are expressed as mean \pm standard deviation, measurement data of multiple groups were compared by variance analysis, and date between two groups were compared by the least significant difference method. $\mathrm{P}<0.05$ was considered statistically significant.

\section{Results}

Comparison of caspase-3 $\mathrm{mRNA}$ and protein levels among the three groups. Fluorescence qPCR was used to analyze the caspase- 3 mRNA levels in the sham, model and treatment groups. The amplification curve is shown in Fig. 1A. The caspase- 3 primers were well designed, and the melting curve had no impurity peak (Fig. 1B). According to the quantitative analysis results presented in Fig. 1C, the caspase-3 mRNA levels in the model and treatment groups were significantly higher than that in the sham group $(\mathrm{P}<0.05)$. However, following treatment with the caspase-3 inhibitor z-DEVD-fmk, the caspase- 3 mRNA level in the treatment group was significantly lower than that in the model group $(\mathrm{P}<0.05)$.

Western blot analysis was used to analyze the caspase- 3 protein expression levels in the brain tissues of the three groups. As shown in Fig. 1C and D, the caspase-3 protein expression level in the model group was significantly higher than that in the sham and treatment groups. Following treating 
Table I. Comparison of neurological impairment scores in the three groups.

\begin{tabular}{|c|c|c|c|c|c|}
\hline \multirow[b]{2}{*}{ Groups } & \multirow[b]{2}{*}{ No. of cases } & \multirow{2}{*}{$\begin{array}{c}\text { Pre-treatment } \\
\text { score }\end{array}$} & \multicolumn{3}{|c|}{ Post-treatment score } \\
\hline & & & $12 \mathrm{~h}$ & $24 \mathrm{~h}$ & $48 \mathrm{~h}$ \\
\hline Sham & 15 & $0.28 \pm 0.22$ & $0.35 \pm 0.41$ & $0.20 \pm 0.20$ & $0.09 \pm 0.03$ \\
\hline Model & 15 & $3.18 \pm 0.91^{\mathrm{a}}$ & $2.87 \pm 0.74^{\mathrm{a}}$ & $2.31 \pm 0.63^{\mathrm{a}}$ & $1.98 \pm 0.51^{\mathrm{a}}$ \\
\hline Treatment & 15 & $3.13 \pm 1.02^{\mathrm{a}, \mathrm{b}}$ & $2.23 \pm 0.59^{\mathrm{a}, \mathrm{b}}$ & $1.57 \pm 0.31^{\mathrm{a}, \mathrm{b}}$ & $0.51 \pm 0.15^{\mathrm{a}, \mathrm{b}}$ \\
\hline
\end{tabular}

${ }^{\mathrm{a}} \mathrm{P}<0.05$ compared with the sham group; ${ }^{\mathrm{b}} \mathrm{P}<0.05$ compared with the treatment group.

A

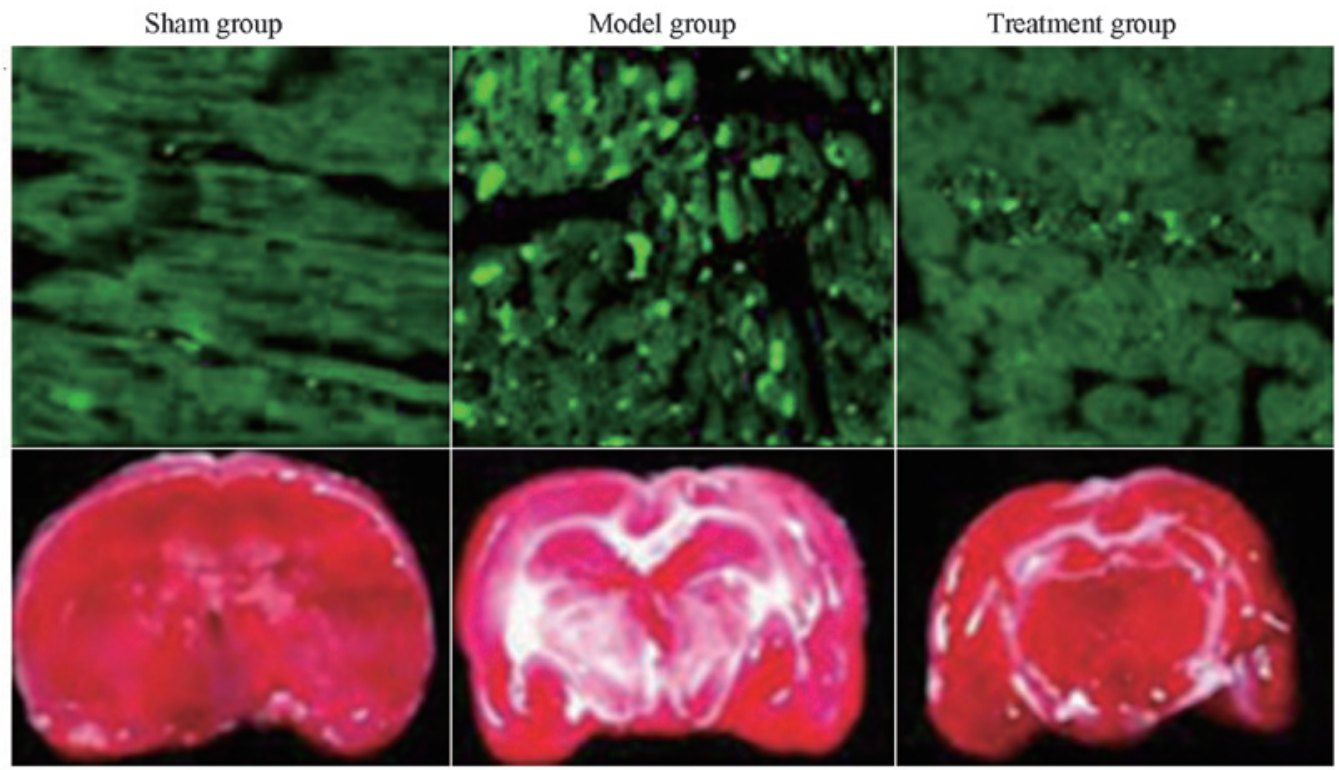

C

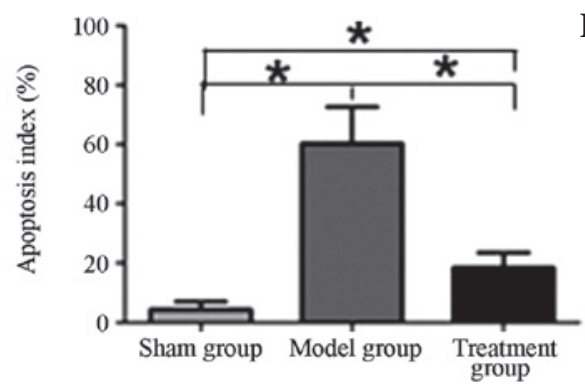

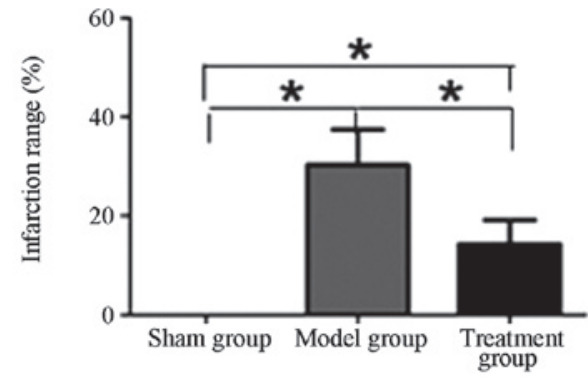

Figure 3. Comparison of apoptosis index and infarction scope in the three groups. (A) TUNEL staining showing apoptosis and (B) TTC staining showing the infarction scope in brain tissue of the sham, model and treatment groups. Quantitative analysis of the (C) apoptosis index as determined by TUNEL staining and (D) infarction scope as determined by TTC staining in brain tissue of the sham, model and treatment groups. TUNEL, terminal deoxynucleotidyl transferase-mediated deoxyuridine triphosphate nick end labeling; TTC, 2,3,5-triphenyltetrazolium chloride.

with the caspase- 3 inhibitor, the caspase-3 protein expression level in the treatment group was significantly decreased compared with that in the model group $(\mathrm{P}<0.05)$, but remained significantly higher than that in the sham group $(\mathrm{P}<0.05)$.

Comparison of caspase- 3 activity in rat brain tissues among the three groups. Firstly, a standard curve for caspase-3 activity was established (Fig. 2A). The standard curve had good linearity $\left(\mathrm{R}^{2}=0.999\right)$. Analysis of the caspase- 3 activity in the rat brain tissues of the three groups indicated that the caspase- 3 activity in the brain tissues of the rats in the model group was significantly higher than that of rats in the sham and treatment groups $(\mathrm{P}<0.05)$. Following treatment with caspase- 3 inhibitor, the caspase- 3 activity in the brain tissues of rats in the treatment group was significantly lower than that of the model group $(\mathrm{P}<0.05)$, but remained higher than that of the sham group $(\mathrm{P}<0.05$; Fig. 2$)$.

Comparison of apoptotic index and infarction scope in rat brain tissues among the three groups. As shown in Fig. 3, the apoptotic index and infarction scope of the rat brain tissues in the model and treatment groups were significantly increased compared with those in the sham group $(\mathrm{P}<0.05)$. Following treatment with caspase- 3 inhibitor, the apoptotic index and 
infarction scope in the treatment group were significantly decreased compared with those in the model group $(\mathrm{P}<0.05)$. However, the apoptotic index and infarction scope of rat brain tissues in the treatment group remained significantly higher than those in the sham group $(\mathrm{P}<0.05)$.

Comparison of the neurological impairment of the three groups. The neurological impairment of the three groups of rats was analyzed 12, 24 and $48 \mathrm{~h}$ after the establishment of the model. As shown in Table I, the neurological impairment scores of the model and treatment groups were significantly increased compared with that of the sham group prior to treatment, $(\mathrm{P}<0.05)$. As the time after treatment increased, the neurological impairment score of the treatment group gradually decreased, and were significantly lower than those in the model group $(\mathrm{P}<0.05)$. However, the neurological impairment scores of the treatment group at the different time-points remained significantly higher than those in the sham group $(\mathrm{P}<0.05)$.

\section{Discussion}

Cell apoptosis, also known as programmed cell death, is an independent death process regulated by genes under physiological or pathological conditions. In the process of acute cerebral infarction, due to cerebral hypoxia and ischemia, brain tissues may undergo necrosis and apoptosis $(14,15)$. Currently, treatments for acute cerebral infarction are focused on the early recanalization of blood flow and saving the reversible ischemic penumbra. Apoptosis is the main form of cell death in the ischemic penumbra; therefore, inhibition of the apoptosis of cells in the ischemic penumbra is the primary method for saving the reversible ischemic penumbra (16). Caspase-3 is a member of the cysteine protease family, and is the major protease that participates in cell apoptosis. It activates the apoptotic pathway by degrading corresponding intracellular substrates, and causes apoptosis; it is therefore known as a molecular switch $(17,18)$. At present, a number of experimental studies have shown that caspase-3 plays an important role in the pathological injury process of myocardial ischemia and cerebral ischemia, and caspase-3 has received great attention due to its role in the final common pathway in apoptosis (19-21).

Since the caspase- 3 protein is affected by the cell surface death receptor-mediated and mitochondrial apoptosis pathways, it plays an important role in mediating the apoptosis of cells (7). On the basis of this, the present study explored the role of caspase- 3 inhibition in modulating caspase- 3 expression, protecting against brain cell apoptosis and maintaining the neurological functions of rats following acute cerebral infarction.

The results showed that the caspase- 3 mRNA and protein levels and enzyme activity increased significantly in the rat brain tissues of an established acute cerebral infarction model, indicating that the apoptosis of brain and nerve cells significantly increases following acute cerebral infarction. This was verified by TUNEL staining, in which quantitative results indicated that the apoptotic index of brain tissues in the model group was significantly increased compared with that in the control group. With the increase of the level of apoptosis, the necrotic scope of the brain tissues in the model group was significantly increased compared with that in the control group, accompanied by a decline of neurological function. When the rats with acute cerebral infarction received caspase-3 inhibitor treatment, it was observed that the caspase-3 mRNA and protein expression levels and enzyme activity in the brain tissue decreased significantly, and the apoptosis index of the brain tissue decreased significantly. Due to a significant reduction in the level of apoptosis, the infarction scope of the brain tissue also significantly declined; the neurological impairment of the rats also significantly declined. This result suggests that the caspase-3 inhibitor z-DEVD-fmk can significantly inhibit the apoptosis of brain tissue cells in rats with acute cerebral infarction, and has a protective effect on brain tissue.

In summary, the caspase-3 inhibitor z-DEVD-fmk significantly reduced the expression of caspase- 3 at the mRNA and protein levels and caspase- 3 activity in the brain tissues of rats with cerebral infarction. In addition, it reduced apoptosis and tissue infarction in the brain tissues and decreased the degree of neurological impairment. Thus, the caspase- 3 inhibitor played a protective role for nervous tissues, and the findings of the present study may act as a reference for the clinical treatment of acute cerebral infarction.

\section{References}

1. Arboix A and Alio J: Acute cardioembolic cerebral infarction: answers to clinical questions. Curr Cardiol Rev 8: 54-67, 2012.

2. Mokin M, Snyder KV, Siddiqui AH, Hopkins LN and Levy EI: Endovascular management and treatment of acute ischemic stroke. Neurosurg Clin N Am 25: 583-592, 2014.

3. Hsieh FI and Chiou HY: Stroke: morbidity, risk factors and care in Taiwan. J Stroke 16: 59-64, 2014.

4. Candelario-Jalil E: Injury and repair mechanisms in ischemic stroke: considerations for the development of novel neurotherapeutics. Curr Opin Investig Drugs 10: 644-654, 2009.

5. Sergeeva SP, Litvickiy PF, Gultyaev MM, Savin AA and Breslavich ID: To the Fas-induced neurons apoptosis mechanisms in stroke pathogenesis. Patol Fiziol Eksp Ter 3: 15-18, 2013 (In Russian).

6. Wang JP, Yang ZT, Liu C, He YH and Zhao SS: L-carnosine inhibits neuronal cell apoptosis through signal transducer and activator of transcription 3 signaling pathway after acute focal cerebral ischemia. Brain Res 1507: 125-133, 2013.

7. Fan W, Dai Y, Xu H, et al: Caspase-3 modulates regenerative response after stroke. Stem Cells 32: 473-486, 2014.

8. Yang B, Ye D and Wang Y: Caspase-3 as a therapeutic target for heart failure. Expert Opin Ther Targets 17: 255-263, 2013.

9. Zhang ZN, Li JY, Zhao Y, Wang JQ, Huang C and Fan GQ: Effects of Tongnao Huoluo acupuncture therapy on Caspase-3 and Bcl-2 of rats with acute cerebral infarction. Zhongguo Zhong Xi Yi Jie He Za Zhi 33: 646-650, 2013 (In Chinese).

10. Rosell A, Cuadrado E, Alvarez-Sabín J, et al: Caspase-3 is related to infarct growth after human ischemic stroke. Neurosci Lett 430: 1-6, 2008.

11. Kikuchi K, Tanaka E, Murai Y and Tancharoen S: Clinical trials in acute ischemic stroke. CNS Drugs 28: 929-938, 2014.

12. Longa EZ, Weinstein PR, Carlson S and Cummins R: Reversible middle cerebral artery occlusion without craniectomy in rats. Stroke 20: 84-91, 1989.

13. Belayev L, Alonso OF, Busto R, Zhao W and Ginsberg MD: Middle cerebral artery occlusion in the rat by intraluminal suture. Neurological and pathological evaluation of an improved model. Stroke 27: 1616-1622, 1996.

14. Elmore S: Apoptosis: A review of programmed cell death. Toxicol Pathol 35: 495-516, 2007.

15. Ouyang L, Shi Z, Zhao S, Wang FT, Zhou TT, Liu B and Bao JK: Programmed cell death pathways in cancer: A review of apoptosis, autophagy and programmed necrosis. Cell Prolif 45: 487-498, 2012. 
16. Oostveen JA, Dunn E, Carter DB and Hall ED: Neuroprotective efficacy and mechanisms of novol pyrrolopyrmidine lipid preoxidation inhibitors in the gerbil forebrain ischemia model. J Gereb BIood FIow Metab 18: 539-547, 1998.

17. Charriaut-Marlangue C: Apoptosis: A target for neuroprotection. Therapie 59: 185-190, 2004.

18. Tanaka K: Pathophysiology of brain injury and targets of treatment in acute ischemic stroke. Rinsho Shinkeigaku 53: 1159-1162, 2013 (In Japanese).

19. Yang B, Ye D and Wang Y: Caspase-3 as a therapeutic target for heart failure. Expert Opin Ther Targets 17: 255-263, 2013.
20. Yu SZ, Yan L, Wang Q, An TL and Guan XQ: Effects of caspase-3 inhibitor on the neuronal apoptosis in rat cerebral cortex after ischemia-reperfusion injury. Zhonghua Bing Li Xue Za Zhi 35: 165-170, 2006 (In Chinese).

21. Ferrer I, Friguls B, Dalfó E, Justicia C and Planas AM: Caspase-dependent and caspase-independent signalling of apoptosis in the penumbra following middle cerebral artery occlusion in the adult rat. Neuropathol Appl Neurobiol 29: 472-481, 2003. 